\title{
Risk Factors for Anastomotic Complications After One-stage Anastomosis for Oesophageal Atresiarisk Factors for Anastomotic Complications After One-stage Anastomosis for Oesophageal Atresia
}

Jinxi Huang

Fujian Children's Hospital https://orcid.org/0000-0002-3178-355X

Songming Hong

fujian children's hospital

Qiang Chen

fujian provincial maternity and children's hospital

Zengchun Wang

fujian provincial maternity and children's hospital

Dianming Wu

fujian provincial maternity and children's hospital

Junjie Hong

fujian children's hospital

Chaoming Zhou ( $\nabla$ sfyzhouchaoming@163.com )

https://orcid.org/0000-0002-3341-9516

Research article

Keywords: anastomotic, oesophageal atresia, Oesophageal

Posted Date: August 3rd, 2020

DOI: https://doi.org/10.21203/rs.3.rs-50683/v1

License: (a) (1) This work is licensed under a Creative Commons Attribution 4.0 International License.

Read Full License 


\section{Abstract}

Objective: The objective of this study is to identify the risk factors for anastomotic complications after one-stage anastomosis for oesophageal atresia.

Methods: A retrospective analysis was performed on the clinical data of 107 children with congenital oesophageal atresia who underwent one-stage anastomosis in our hospital from January 2016 to December 2018. Single-factor and multivariate logistic regression analyses were performed to determine the risk factors for anastomotic fistula and anastomotic stenosis.

Results: A total of 107 children with oesophageal atresia underwent one-stage anastomosis, and the incidence of anastomotic fistula was $26.2 \%$. The probability of anastomotic stenosis in the long term was $52.3 \%$, and the incidence of refractory stenosis (dilation $\geq 5$ times) was $13.1 \%$. Analysis of the clinical count data in the anastomotic fistula group and non-anastomotic fistula group showed that preoperative albumin $(F=4.199, P=0.043)$, low birth weight $(F=7.668, P=0.007)$ and long gap defects $(\mathrm{F}=6.107, \mathrm{P}=0.015)$ were risk factors for postoperative anastomotic fistula. Further multivariate logistic regression analysis showed that low birth weight (Wald2 $=4.499, P=0.034, \mathrm{OR}=2.775)$ and long gap defects (Wald2 $=6.769, \mathrm{P}=0.009, \mathrm{OR}=4.939$ ) were independent risk factors for postoperative anastomotic fistula. Premature delivery $(F=5.338, P=0.023)$, anastomotic fistula $(F=11.381, P=0.001)$, endoscopic surgery $(F=6.343, P=0.013)$, preoperative neutrophil count $(F=8.602, P=0.004)$, preoperative low albumin $(F=8.410, P=0.005)$, and a preoperative prognostic nutritional index $<54(F=5.54, P=0.02)$ were risk factors for long-term anastomotic stenosis in children. Further multivariate logistic regression analysis showed that postoperative anastomotic fistula (Wald2 $=11.417, \mathrm{P}=0.001, \mathrm{OR}=8.798$ ), endoscopic surgery (Wald2=9.633, $P=0.002, \mathrm{OR}=4.808$ ), and a prognostic nutritional index $<54$ (Wald2=4.540, $P=0.002$, $\mathrm{OR}=2.3798$ ) were independent risk factors for long-term anastomotic stenosis.

Conclusion: Low birth weight and long gap defects are important predictors of postoperative anastomotic fistula, and the possibility of long-term anastomotic stenosis should be considered. The long-term risk of anastomotic stenosis was increased in children undergoing endoscopic surgery and in those with a preoperative prognostic nutritional index $<54$.

\section{Background}

Oesophageal atresia (EA) is a congenital malformation of the oesophagus and a serious malformation of the digestive tract. The global overall incidence is 2.4 cases per 100,000 , and the incidence of congenital EA in China is 1/1250-1/4000 [1]. At present, this disease is believed to be due to the interruption of oesophageal continuity caused by abnormal development of the preembryonic intestinal area.

Approximately $70-90 \%$ of children often have tracheal oesophageal fistula (TEF) [2], and $55 \%$ of children may have other systemic congenital malformations, especially severe heart malformations, which is an important factor for poor prognosis in children [3]. The treatment of EA has made great progress in the past 20 years, and the survival rate can reach more than $90 \%$. With the development of endoscopic 
technology in recent years, video-assisted one-stage anastomosis has gradually become the main surgical method instead of open-chest operations.

Common EA postoperative complications include acute respiratory failure, pneumonia, anastomotic fistula, anastomotic stenosis, tracheal stenosis, gastroesophageal reflux and eosinophilic oesophagitis [4]. Anastomotic complications mainly include recent postoperative anastomotic fistula and long-term anastomotic stenosis, and anastomotic fistula is one of the important causes of postoperative death. Severe anastomotic fistula may increase the risk of long-term anastomotic stenosis or even refractory stenosis [5]. This study attempts to explore the related risk factors for postoperative anastomotic complications in children with congenital EA through a retrospective study.

\section{Methods}

Data from the 127 children born in or admitted to our hospital with EA were collected and followed, with 14 of the parents of sick children refusing surgery. A total of 113 children received surgical treatment, and 6 died perioperatively; thus, a total of 107 children were enrolled. The patients were divided into two groups according to the occurrence of anastomotic fistula.

\subsection{General conditions}

From January 2013 to December 2018, 107 children with EA underwent surgery in our hospital with complete clinical and pathological data; there were 74 male patients and 33 female patients. The data of sex, birth weight, gestational age, surgical method, defect length (intraoperative measurement), preoperative mechanical ventilation, and combined deformity were collected (Table 1).

\subsection{Preoperative data}

1) Serological indicators: the preoperative neutrophil count, preoperative lymphocyte count, and neutrophil to lymphocyte ratio (NLR).

2) Nutritional indicators: the preoperative albumin, prognostic nutritional index (PNI) and albumin to globulin ratio. $\mathrm{PNI}=10 \mathrm{ALB}(\mathrm{dg} / \mathrm{L})+5 * \mathrm{LYMPH} \#$

3) Postoperative data: anastomotic fistula, anastomotic stenosis, pulmonary infection, oesophageal dilatation times and first dilatation time.

1.3 Inclusion criteria: children born or admitted to our hospital with congenital EA and receiving stage I anastomosis with complete clinical data. The exclusion criteria were as follows: 1. parents refused the operation; 2. patients who could not undergo delayed anastomosis in the first stage of the operation; and 3. patients who died in the perioperative period.

1.4 SPSS 22.0 software was used to analyse the data, and univariate and multivariate logistic regression analyses were performed for EA anastomotic fistula and anastomotic stenosis. The odds ratio (OR) and 
95\% confidence interval $(95 \% \mathrm{Cl})$ were used to indicate the correlation. T tests were used for the comparison of oesophageal dilatation analysis results, and $\mathrm{P}<0.05$ was considered statistically significant.

\section{Results}

\subsection{Recent anastomotic complications: anastomotic fistula}

Anastomotic fistula is a common and dangerous recent anastomotic complication of oesophageal reconstruction. In this study, the incidence of anastomotic fistula was $26.3 \%$, which is slightly higher than the $20 \%$ reported in other studies [6]. The occurrence of anastomotic fistula after anastomosis in adults is mainly related to anastomotic tension and anastomotic blood supply [7]. It is difficult to quantitatively evaluate anastomotic tension and anastomotic blood supply during neonatal EA stage I anastomosis. In this study, clinical enumeration data were collected to seek objective clinical indicators to assess the risk of anastomotic complications. With the analysis of two groups of data, continuous variables using single-factor analysis of variance and classification variables using the chi-square test, the study found that preoperative albumin, birth weight and defect length were postoperative anastomotic fistula-related factors. The differences between groups were statistically significant, and the time, gestational age, sex, preoperative operation method of mechanical ventilation, neutrophil count, lymphocyte count, NLR, PNI, albumin, globulin, and merged heart deformities were not significantly different between these factors in the two groups. Further multivariate logistic regression analysis showed that a birth weight less than $2500 \mathrm{~g}$ and long gap defects larger than $3 \mathrm{~cm}$ were independent risk factors for postoperative anastomotic fistula in children.(Table $2 \&$ Table 3 )

\subsection{Long-term anastomotic complications: anastomotic stenosis}

Anastomotic stricture: symptoms of dysphagia and anastomotic stricture were confirmed by gastrointestinal radiography or endoscopy. This study found that the surgical method, gestational age, preoperative neutrophil count, $\mathrm{PNI}$ and anastomotic fistula may be related factors for the occurrence of anastomotic stenosis. Further multivariate logistic regression analysis found that open surgery, anastomotic fistula and low nutritional status ( $\mathrm{PNI} \leq 54)$ were high-risk factors for long-term anastomotic stenosis.(Table 4 \& Table 5)

\section{Discussion}

1. Risk factor analysis of recent anastomotic fistula

The occurrence of anastomotic fistula after first-stage oesophagostomy in children with congenital EA is still a serious complication that troubles surgeons and has an important influence on the prognosis and quality of life of children[8]. The occurrence of anastomotic fistula is mainly related to anastomotic blood supply, anastomotic tension and other factors $[9,10]$. The incidence of anastomotic fistula after end-to-end oesophagostomy is reported to be approximately $8-12 \%$ in adults[11], and the incidence of anastomotic 
fistula after EA is approximately $20 \%[3]$. This may be related to the large anastomotic tension and poor anastomotic blood supply caused by oesophageal defects. In this study, univariate analysis found that albumin, defect length and birth weight may be related to the occurrence of anastomotic fistula. Multivariate logistic regression analysis found that long gap defects and low birth weight were high risk factors for anastomotic fistula.

Preoperative low protein may lead to postoperative anastomotic oedema patients, which increases anastomotic tension and leads to poor local healing $[12,13]$. However, in multiple factor analysis, albumin levels were not included in the regression equation. This may be because, in this study, the number of patients with low albumin (albumin $<28 \mathrm{~g} / \mathrm{L}$ ) was less relevant, further expanding the sample size to find the best values for a more suitable cut-off value to evaluate the relationship between albumin levels and anastomotic fistula.

The defect length is one of the important indexes for the preoperative evaluation of operation difficulty in children with EA[14]. In this study, the mean defect length was $1.53 \pm 0.96 \mathrm{~cm}, 1.91 \pm 1.01 \mathrm{~cm}$ in children with anastomotic fistula, and $1.39 \pm 0.91 \mathrm{~cm}$ in children without anastomotic fistula, showing statistically significant differences. There were 14 patients with long gap EA, and the possibility of anastomotic fistula was 4.9 times that of non-long-gap EA. Long defects are an independent risk factor for anastomotic fistula. Children with an intraoperative defect length $>3 \mathrm{~cm}$ were more prone to anastomotic fistula 7 to 10 days after surgery. Four cases were excluded from this study due to intrathoracic extension and delayed anastomosis of long gap EAs. fourteen cases of long gap EAs appeared among 8 cases of severe anastomotic fistula. We believe that the use of long gap defects to extend the anastomosis may be a better choice.

Birth weight reflects the developmental level of children in utero, and a low birth weight is often associated with a history of premature birth and foetal distress [15]. Children with a low birth weight may have more oesophageal defects due to the short duration of natural extension of the oesophagus in utero, and these defects may be accompanied by poorer peripheral circulation and lower cardiac output [16], which may lead to poor local blood supply at the anastomosis. The counting data showed that 8 of 32 children with a low birth weight had heart malformations, while only 6 of 75 children with a normal weight had heart malformations. This study found that the risk of anastomotic fistula was 2.7 times higher in children with a low birth weight than in children with a normal weight.

\section{Risk factor analysis of long-term anastomotic stenosis}

In this study, the probability of anastomotic stenosis was $52.3 \%$, and the risk of anastomotic stenosis factors, including anastomotic fistula, long defects, severe gastroesophageal reflux and eosinophilic oesophagitis [17] [18], may cause inflammation of the anastomotic fistula after conservative treatment as well as related scar factors. Anastomotic fistula was one of the most relevant factors, $\mathrm{OR}=7.506$, but the length of the defect was not included in the regression equation. Univariate analysis revealed that the surgical method, gestational age, preoperative neutrophil count, and PNI may also be related factors. In multivariate analysis, thoracoscopic surgery, anastomotic fistula, and low nutritional status (PNI $\leq 54)$ 
were high risk factors for anastomotic stenosis. The incidence of anastomotic stenosis after thoracoscopic surgery was $32 / 49$, which was significantly higher than that after open surgery $(24 / 58)$. Open surgery may provide better oesophageal mucosal alignment and firmer end-to-end anastomosis. Thoracoscopic oesophageal anastomosis is still under development, and it is believed that the incidence of anastomotic stenosis in endoscopic surgery will decrease with the maturity of endoscopic technology in the future. According to the results of this study, thoracoscopic surgery is still considered to increase the risk of anastomotic stenosis.

Most anastomotic fistulas can be cured by conservative treatment, including total parenteral nutrition support or post-pyloric enteral nutrition support, pleural drainage and adequate antibiotic use $[19,20]$. Some scholars advocate early operations to close the fistula in order to avoid severe pulmonary infection and empyema $[21,22]$. Anastomotic fistula leads to severe anastomotic inflammation. Although conservative treatment is successful, it is still inevitable that the incidence of anastomotic stenosis will increase or even develop into refractory anastomotic stenosis due to scarring [23].

At present, there are few studies on nutritional assessment indicators for neonates, and the PNI is a commonly used nutritional assessment indicator for gastrointestinal tumours in adults [24]. Univariate analysis found that the preoperative difference in PNI values between the two groups was statistically significant. At present, there is no clear numerical classification of neonatal nutritional risk with the PNI. By calculating the Youden index, we obtained the optimal truncation value of the PNI value in this sample to be 54. Based on this, we conducted a multifactor analysis and found that the risk of anastomotic stenosis in children in the low PNI group (PNI $\leq 54)$ was 5.7 times higher than that in the high PNI group. For children with preoperative nutritional risk, perioperative nutritional support may help reduce the risk of long-term anastomotic stenosis, which needs to be further confirmed by future prospective studies.

\section{Conclusion}

Low birth weight and long gap defects are important predictors of postoperative anastomotic fistula, and the possibility of long-term anastomotic stenosis should be considered. The long-term risk of anastomotic stenosis was increased in children undergoing endoscopic surgery and in those with a preoperative prognostic nutritional index $<54$.

Table 1 General Information 


\begin{tabular}{|ll|}
\hline Information types & Value \\
\hline Gender (male/female) & $74 / 33$ \\
Gestational weeks & $38.51 \pm 1.61$ \\
Birth weight (kg) & $2.83 \pm 0.48$ \\
Operation method (endoscopic/open) & $49 / 58$ \\
Preoperative mechanical ventilation (yes/no) & $7 / 100$ \\
Deformity (yes/no) & $33 / 74$ \\
Cardiovascular anomalies & 14 \\
Gastrointestinal anomalies & 8 \\
Genitourinary anomalies anomalies & 4 \\
Musculoskeletal anomalies & 11 \\
Respiratory anomalies & 9 \\
Other anomalies & 5 \\
Albumin (g/L) & $34.16 \pm 3.2$ \\
Neutrophil (10*9/ L) & $9.97 \pm 4.89$ \\
lymphocyte (10*9/ L) & $3.5 \pm 1.52$ \\
NLR & $3.28 \pm 1.88$ \\
Albumin/Globulin & $1.87 \pm 0.71$ \\
PNI & $51.69 \pm 8.46$ \\
Gap \cm $\square$ & $1.53 \pm 0.96$ \\
Anastomotic fistula & 28 \\
Anastomotic stenosis & 56 \\
\hline
\end{tabular}

Table 2 Comparison of complications between anastomotic fistula group and non-anastomotic fistula group 


\begin{tabular}{|c|c|c|c|c|c|}
\hline Factors & & $\begin{array}{l}\text { Anastomotic } \\
\text { fistula }\end{array}$ & $\begin{array}{l}\text { Non-anastomotic } \\
\text { fistula }\end{array}$ & $\mathrm{F} / \otimes 2$ & $P$ \\
\hline Gestational weeks & & $38.61 \pm 1.61$ & $38.32 \pm 1.63$ & 0.651 & 0.421 \\
\hline Birth weight (kg) & & $2.62 \pm 0.51$ & $2.90 \pm 0.47$ & 7.668 & 0.007 \\
\hline Gap $₫ \mathrm{~cm} \rrbracket$ & & $1.91 \pm 1.01$ & $1.39 \pm 0.91$ & 6.107 & 0.015 \\
\hline Albumin $(\mathrm{g} / \mathrm{L})$ & & $33.11 \pm 3.04$ & $34.54 \pm 3.19$ & 4.199 & 0.043 \\
\hline Neutrophil $(10 \star 9 / L)$ & & $10.29 \pm 5.34$ & $9.85 \pm 4.75$ & 0.174 & 0.678 \\
\hline Lymphocyte(10*9/L) & & $3.69 \pm 1.57$ & $3.44 \pm 1.50$ & 0.583 & 0.447 \\
\hline NLR & & $3.13 \pm 1.89$ & $3.30 \pm 1.88$ & 0.15 & 0.7 \\
\hline PNI & & $51.59 \pm 7.67$ & $51.73 \pm 8.78$ & 0.006 & 0.938 \\
\hline Albumin/Globulin & & $2.03 \pm 1.23$ & $1.81 \pm 0.38$ & 2.026 & 0.158 \\
\hline \multirow[t]{2}{*}{ Deformity } & Yes & 12 & 21 & & \\
\hline & No & 16 & 58 & 2.567 & 0.088 \\
\hline \multirow[t]{2}{*}{ Cardiovascular anomalies } & Yes & 4 & 10 & & \\
\hline & No & 24 & 69 & 0.635 & 0.316 \\
\hline \multirow{2}{*}{$\begin{array}{l}\text { Preoperative mechanical } \\
\text { ventilation }\end{array}$} & Yes & 1 & 6 & & \\
\hline & No & 27 & 73 & 0.547 & 0.41 \\
\hline \multirow[t]{2}{*}{ Gender } & Male & 18 & 56 & & \\
\hline & Female & 10 & 23 & 0.422 & 0.336 \\
\hline \multirow[t]{2}{*}{ Operation method } & Open & 15 & 43 & & \\
\hline & Endoscopic & 13 & 36 & 0.006 & 0.055 \\
\hline
\end{tabular}

Table 3 Multiple factor logistic regression

\begin{tabular}{|llll|}
\hline Factors & Wald $₫ 2$ & P values & OR \\
\hline Low birth weight & 4.499 & 0.004 & 2.775 \\
\hline long gap atresia & 6.769 & 0.034 & 4.939 \\
\hline
\end{tabular}

Table 4 Univariate analysis of anastomotic stenosis 


\begin{tabular}{|c|c|c|c|c|c|}
\hline Factors & & $\begin{array}{l}\text { Anastomotic } \\
\text { stenosis }\end{array}$ & $\begin{array}{l}\text { Non-anastomotic } \\
\text { stenosis }\end{array}$ & 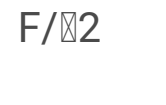 & $P$ \\
\hline Gestational weeks & & $38.20 \pm 1.73$ & $38.90 \pm 1.39$ & 5.338 & 0.023 \\
\hline Birth weight (kg) & & $2.77 \pm 0.52$ & $2.90 \pm 0.44$ & 1.882 & 0.173 \\
\hline Gap $₫ \mathrm{~cm} \rrbracket$ & & $1.60 \pm 0.93$ & $1.46 \pm 0.99$ & 0.546 & 0.462 \\
\hline Neutrophil $(10 \star 9 / L)$ & & $8.69 \pm 4.69$ & $11.37 \pm 4.77$ & 8.602 & 0.004 \\
\hline Lymphocyte (10*9/L) & & $3.31 \pm 1.28$ & $3.72 \pm 1.73$ & 1.947 & 0.166 \\
\hline NLR & & $3.00 \pm 2.05$ & $3.53 \pm 1.64$ & 2.097 & 0.151 \\
\hline PNI & & $49.9 \pm 6.97$ & $53.67 \pm 9.53$ & 5.54 & 0.02 \\
\hline Albumin/Globulin & & $1.77 \pm 0.35$ & $1.98 \pm 0.95$ & 2.584 & 0.112 \\
\hline \multirow[t]{2}{*}{ Deformity } & Yes & 17 & 16 & & \\
\hline & No & 39 & 35 & 0.013 & 0.538 \\
\hline \multirow[t]{2}{*}{ Cardiovascular anomalies } & Yes & 8 & 6 & & \\
\hline & No & 48 & 45 & 0.149 & 0.462 \\
\hline \multirow{2}{*}{$\begin{array}{l}\text { Preoperative mechanical } \\
\text { ventilation }\end{array}$} & Yes & 4 & 3 & & \\
\hline & No & 52 & 48 & 0.69 & 0.552 \\
\hline \multirow[t]{2}{*}{ Gender } & Male & 39 & 35 & & \\
\hline & Female & 17 & 16 & 0.013 & 0.538 \\
\hline \multirow[t]{2}{*}{ Anastomotic fistula } & Yes & 22 & 6 & & \\
\hline & No & 34 & 45 & 10.464 & 0.001 \\
\hline \multirow[t]{2}{*}{ Operation method } & Open & 24 & 34 & & \\
\hline & Endoscopic & 32 & 17 & 6.096 & 0.013 \\
\hline
\end{tabular}

Table 5. Logistic regression analysis Results

\begin{tabular}{|llll|}
\hline Factors & Wald $₫ 2$ & P values & ORヌ95\%Cl \\
\hline Open operation & 5.106 & 0.025 & $2.993 \rrbracket 1.147-7.815 \rrbracket$ \\
\hline Anastomotic fistula & 10.204 & 0.01 & $7.506 \rrbracket 2.179-25.856 \rrbracket$ \\
\hline PNI $\leq 54$ & 7.829 & 0.005 & $5.725 \rrbracket 1.686-19.434 \rrbracket$ \\
\hline
\end{tabular}




\section{Abbreviations}

EA: Oesophageal atresia,TEF:tracheal oesophageal fistula, NLR:neutrophil to lymphocyte ratio, PNI:prognostic nutritional index, OR:odds ratio, Cl:confidence interval.

\section{Declarations}

\Ethics approval and consent to participate:The hospital ethics committee approval was granted of this article.

Q Consent for publication was obtained from the child's parents.

$\otimes$ Availability of data and materials: Written informed consent was obtained from the patient for publication of this article.

\Competing interests: The authors declare that they have no competing interests.

\ Funding: no funding.

Q Authors' contributions:Jinxi Huang participated in clinical practice, contributed to collection and analysis of data, drafting the manuscript, and revising it. Junjie Hong carried out data collection. Qiang Chen participated in clinical practice. Dianming Wu and Songming Hong helped in design of study and drafting the manuscript. Chaoming Zhou carried out patient recruitment and clinical practice, contributed to conception, design, drafting the manuscript, and revising it. All authors read and approved the final manuscript.

\section{References}

1. Pedersen R N , Calzolari E , Husby S, et al. Oesophageal atresia: prevalence, prenatal diagnosis and associated anomalies in 23 European regions[J]. Archives of Disease in Childhood, 2012, 97(3):227232.

2. Bogs Thomas,Zwink Nadine,Chonitzki Vera et al. Esophageal Atresia with or without Tracheoesophageal Fistula (EA/TEF): Association of Different EA/TEF Subtypes with Specific Cooccurring Congenital Anomalies and Implications for Diagnostic Workup.[J] .Eur J Pediatr Surg, 2018, 28: 176-182.

3. van Lennep Marinde,Singendonk Maartje M J,Dall'Oglio Luigi et al. Oesophageal atresia.[J] .Nat Rev Dis Primers, 2019, 5: 26. 
4. R Daum. Postoperative complications following operation for oesophageal atresia and tracheooesophageal fistula[J]. progress in pediatric surgery, 1970, 1(1):209.

5. Baird Robert,Laberge Jean-Martin,Lévesque Dominique,Anastomotic stricture after esophageal atresia repair: a critical review of recent literature.[J] .Eur J Pediatr Surg, 2013, 23: 204-13.

6. Reusens Helena,Matthyssens Lucas,Vercauteren Charlotte et al. Multicentre survey on the current surgical management of oesophageal atresia in Belgium and Luxembourg.[J] .J. Pediatr. Surg., 2017, 52: 239-246.

7. Korenaga D,Toh Y,Maekawa S et al. Intra-operative measurement of the tissue blood flow for evaluating blood supply to the gastric tube for esophageal reconstruction.[J] .Hepatogastroenterology, 1998, 45: 2179-80.

8. Legrand Céline,Michaud Laurent,Salleron Julia et al. Long-term outcome of children with oesophageal atresia type III.[J] .Arch. Dis. Child., 2012, 97: 808-11.

9. Åkesson Oscar,Falkenback Dan,Johansson Göran et al. Surface Microdialysis Detects Ischemia After Esophageal Resection-An Experimental Animal Study.[J] .J. Surg. Res., 2020, 245: 537-543.

10. Lyall P,Bao-Quan Q,Beasley $S$, The effect of neck flexion on oesophageal tension in the pig and its relevance to repaired oesophageal atresia.[J] .Pediatr. Surg. Int., 2001, 17: 193-5.

11. Turkyilmaz A,Eroglu A,Aydin $Y$ et al. The management of esophagogastric anastomotic leak after esophagectomy for esophageal carcinoma.[J] .Dis. Esophagus, 2009, 22: 119-26.

12. Huang Jinxi,Zhou Yi,Wang Chenghu et al. Logistic regression analysis of the risk factors of anastomotic fistula after radical resection of esophageal-cardiac cancer.[J] .Thorac Cancer, 2017, 8: 666-671.

13. Kudsk Kenneth A,Tolley Elizabeth A,DeWitt R Chance et al. Preoperative albumin and surgical site identify surgical risk for major postoperative complications.[J] .JPEN J Parenter Enteral Nutr, 2003, 27: 1-9.

14. Stadil T, Koivusalo A, Svensson J F, et al. Surgical treatment and major complications within the first year of life in newborns with long gap esophageal atresia gross type A and B - a systematic review. [J]. Journal of Pediatric Surgery, 2019, 54(11): 2242-2249.

15. K C Anil,Basel Prem Lal,Singh Sarswoti,Low birth weight and its associated risk factors: Health facility-based case-control study.[J] .PLoS ONE, 2020, 15: e0234907.

16. Kinoshita Meredith,Hawkes Colin Patrick,Ryan C Anthony et al. Perfusion index in the very preterm infant.[J] .Acta Paediatr., 2013, 102: e398-401.

17. Okata Yuichi,Maeda Kosaku,Bitoh Yuko et al. Evaluation of the intraoperative risk factors for esophageal anastomotic complications after primary repair of esophageal atresia with tracheoesophageal fistula.[J] .Pediatr. Surg. Int., 2016, 32: 869-73.

18. Shah R,Varjavandi V,Krishnan U,Predictive factors for complications in children with esophageal atresia and tracheoesophageal fistula.[J] .Dis. Esophagus, 2015, 28: 216-23. 
19. Cui Xu,He Yuanbin,Chen Liu et al. The Value of Thoracic Lavage in the Treatment of Anastomotic Leakage After Surgery for Type III Esophageal Atresia.[J] .Med. Sci. Monit., 2020, 26: e919962.

20. D'Urzo C , Buonuomo V , Rando G , et al. Major anastomotic dehiscence after repair of esophageal atresia: Conservative management or reoperation?[J]. Diseases of the Esophagus, 2005, 18.

21. Godoy Jorge,Ferraris Tomas,Guelfand Miguel,Early Management of Esophageal Leak in Esophageal Atresia: Changing Paradigms.[J] .J Laparoendosc Adv Surg Tech A, 2020, undefined: undefined.

22. Cano Novillo Indalecio,Aneiros Castro Belén,Godoy Lenz Jorge et al. Thoracoscopic approach for complications after esophageal atresia repair: initial experience.[J].Asian J Endosc Surg, 2020, 13: 147-151.

23. Vergouwe Floor W T,Vlot John,IJsselstijn Hanneke et al. Risk factors for refractory anastomotic strictures after oesophageal atresia repair: a multicentre study.[J] .Arch. Dis. Child., 2019, 104: 152157.

24. Okadome Kazuo,Baba Yoshifumi,Yagi Taisuke et al. Prognostic Nutritional Index, Tumor-infiltrating Lymphocytes, and Prognosis in Patients with Esophageal Cancer.[J] .Ann. Surg., 2020, 271: 693-700. 